\title{
Uncertainty Management in History Matching and Forecasting a Field
}

\section{Al Muatasim Al Bahlani, Petroleum Development Oman}

South of Oman is rich of many heavy oil fields which contain multi billion barrels of heavy oil. However, these fields come with a sharp contrast in their accumulative and flow reservoir characteristics. Thus, the range of uncertainties is wide and an educated method should be developed to manage them. The following presentation is a showcase of the process used to reduce the associated uncertainties with modeling such fields mainly for forecasting and history matching. Such methods were used to decide on pattern development and observation wells locations. The method used is evolving as more data is acquired along the projects life. 